\title{
A MULTI-POINT MULTI-OBJECTIVE TRANSPORTATION ROUTE-OPTIMIZATION MODEL OF HAZARDOUS CHEMICALS
}

\author{
JINGNA LI ${ }^{1}$, JINGHONG WANG $^{1}$, YOURAN ZHI ${ }^{2} \&$ XUHAI PAN ${ }^{1}$ \\ ${ }^{1}$ Jiangsu Key Laboratory of Hazardous Chemicals Safety and Control, College of Safety Science and Engineering, \\ Nanjing Tech University, China \\ ${ }^{2}$ College of Mechanical Engineering, Nanjing Institute of Technology, China
}

\begin{abstract}
The transportation of dangerous goods/hazardous chemicals is a hot issue in the area of industrial safety. This paper investigates route optimization for the transportation of Adipic acid based on the fuzzy compromise approach and the iterative algorithm, proposing a multi-point multi-objective decisionmaking model of road transportation routing with minimum transportation risk, minimum transportation cost and minimum sensitive population. The three objective functions are integrated with weights by path optimization and risk decision model. The optimal compromised solution is obtained using the extended label correcting method. The iterative algorithm is used to remove the road section with maximum risk and get the final optimized route. The final optimized route is obtained by multiple optimizations, which combines the dual advantages of extended label correcting method and the iterative algorithm. Compared with the optimized route obtained by only using one-off optimization, the proposed method can consider the weight of the multi-objective functions to better balance the relationship among different objectives and is better to meet the requirement of the government, the transporters and the general public. An example is used to demonstrate the model. Using the route optimization model proposed in this paper, it is feasible to acquire a good balance between the risks and benefits associated with hazardous chemical transportation.
\end{abstract}

Keywords: hazardous chemical transportation, route optimization, extended label correcting, multipoint multi-objective, transportation risk, transportation cost, sensitive population.

\section{INTRODUCTION}

1,6-Hexanediaminere produced and transported by chemical enterprises, and these chemicals can cause damage if improperly transported [1]. Hazardous materials transportation constitutes a serious safety problem and growing concern at local, national, and international levels [2], [3]. The number of dangerous chemicals in an industrial park is very large, and it is common for a variety of dangerous chemicals to be transported concurrently. In the course of transport, this means of shipping can lead to a chain reaction, with the result that the surrounding chemical plants and residents suffer a disaster and great damage.

Route optimization of hazardous chemicals can be divided into the problem of singleobjective and multi-objective functions, and the primary algorithms include the Dijkstra algorithm and the Ford algorithm. During the process of model building, TavakkoliMoghaddam et al. [4] took the two goals of time and vehicle capacity into account concurrently. Frank et al. [5] built a model of risk constrained on the foundation of the contradiction between economic efficiency and population security. Clegg et al. [6] outlined a multi-modal, elastic, equilibrium transportation model in which signal green-times and prices charged to traverse a route were considered. Samanlioglu [7] proposed a new multi-objective location-routing model considering three criteria: minimizing total cost, minimizing total transportation risk, and minimizing total risk for the population around treatment and disposal centers. In recent years, scholars began to study the hazardous chemicals transportation path utilizing networks. These researchers restrict the network to a tree and remove the second 
level. The researchers later develop a simple construction heuristic to expand the solution of the tree design problem by adding road segments. Erkut and Gzara [8] proposed a two-layer model, which minimized risk in the upper and the lowest cost in the lower. Bianco et al. [9] proposed a risk equilibrium theory for the problem that the regional risk balance is not considered globally, which minimized total risk of the road network. Kara and Verter [10] are the first people to pose the hazmat network design problem as a bilevel problem. These authors formulate the design problem as a bilevel integer programming model, where hazmats are grouped into categories based on risk impact, and a network is designed for each group.

At present, most scholars have studied the two-tier planning model and the basic optimization route method. However, the existing research considered little about a variety of hazardous chemicals with three or more transport objectives. In this case, there are several differences in the path selection method. Therefore, in this paper, we consider a situation where various kinds of 1,6-Hexanediaminere transported at the same time. According to the factors that influence the objective function and the relevant literature, we build an mathematical model as well as propose a comprehensive algorithm to search the optimization route. Finally, a case study is used to verify the proposed algorithm.

\section{MATHEMATICAL MODEL}

\subsection{Establishment of the optimization index system}

The objective of defining the transportation route for hazardous chemicals is the prerequisite for ensuring safe transportation routes. The characteristics of transportation routes determine the specificity of the transportation process. The transportation characteristics of hazardous chemicals include [11], [12]:

- Hazardous chemicals have a higher probability of accidents and increased transportation risks.

- 1,6-Hexanediaminere a source of dangerous traffic. These goods should be delivered to the destination as soon as possible, and potential threats along the route should be eliminated.

- Hazardous chemicals transportation accidents have a significant impact on the surrounding personnel, including a large number of toxic gas leaks, explosions, and fires.

- Hazardous chemicals transportation accidents have characteristics that include sudden, delay, long-term and sociality effects.

Based on the above characteristics, this paper considers minimizing the transportation risk, transportation costs and the sensitive population to establish the multi-objective decision model. In this paper, we use the principle of safety economics to turn the single objective into multi-objective [12]. At the same time, for multi-point hazardous chemicals road transportation, if too many hazardous chemicals vehicles are passed on the road, the transportation risk of the road will increase. Therefore, we use an iterative algorithm to optimize the transportation path and reduce the transportation risks.

Assume the total transport objective is $W$, the transport risk is $W 1$, the transportation cost is $W 2$, and the number of sensitive population is W3. According to the principle of index systems, by combining the actual situation, safety requirements, and relevant literature, this paper determines the impact factors of $W 1, W 2$ and $W 3$. The main factors are shown in Fig. 1. 


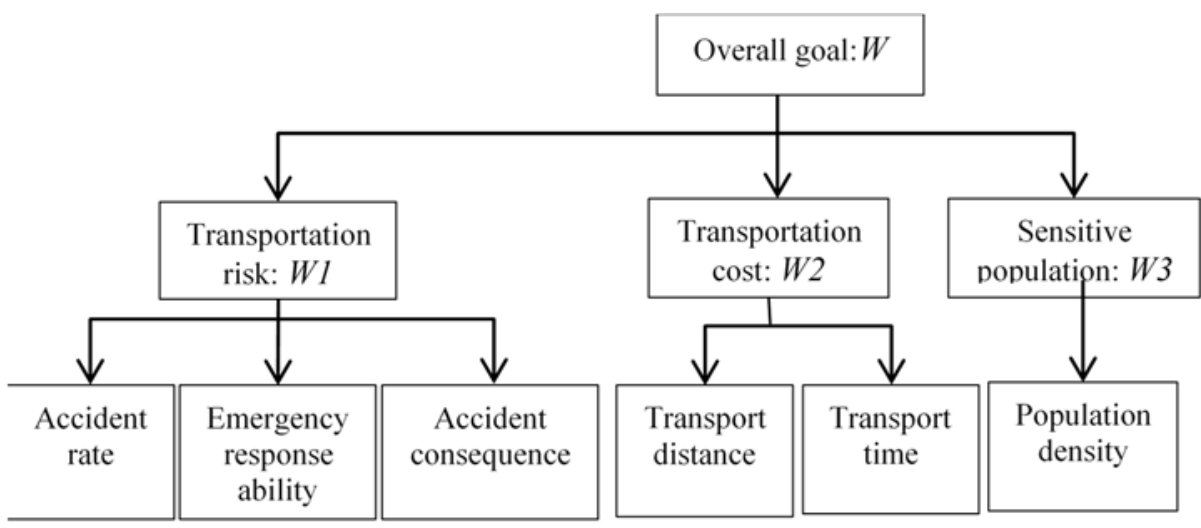

Figure 1: Influencing factors of the overall goal.

\subsection{Model formulation}

Given the network $G=(V, E), V$ is the set of nodes and $E$ is the set of tangential arcs between nodes, the node represents transport point and the arc represents road. $V=n, E=m$. For each $\operatorname{arc}(i, j)$ in graph $G, i, j \in V, i$ is the start point and $j$ is the end point. Any arc $(i, j) \in E$ has a three-dimensional objective vector $r_{(i, j)}=\left(r_{1(i, j)}, r_{2(i, j)}, r_{3(i, j)}\right) . r_{1(i, j)}, r_{2(i, j)}, r_{3(i, j)}$ respectively represent the transportation risk, the transportation cost and the number of sensitive objectives people of arc $(i, j)$. The optimization of the hazardous chemicals road transportation path chooses a path where transportation risk, transportation costs, and the number of sensitive objectives are best for transporters and social groups. The variables are defined as follows:

$$
X_{i j}=\left\{\begin{array}{cc}
1, & \text { transportation vehicle through the road }(\mathrm{i}, \mathrm{j}) . \\
0, & \text { otherwise. }
\end{array}\right.
$$

$$
\mathrm{Y}_{i j}= \begin{cases}1, & \text { dangerous goods are permitted to transport on the road }(\mathrm{i}, \mathrm{j}) \text { by government. } \\ 0, & \text { otherwise. }\end{cases}
$$

Goal 1: The minimization of accidental risk:

Set the risk of each arc as $r_{1(i, j)}, r_{1}(i, j)=C_{i j} h_{i j} P_{i j} X_{i j} Y_{i j}$, there are

$$
\mathrm{W} 1=\min \sum_{i=1}^{n} \sum_{j=1}^{n} r_{1}(i, j) .
$$

Goal 2: The minimization of transportation costs:

Set the cost of each arc as $r_{2(i, j)}, r_{2}(i, j)=d_{i j} / v$, there are

$$
\mathrm{W} 2=\min \sum_{i=1}^{n} \sum_{j=1}^{n} r_{2}(i, j) .
$$

Goal 3: The minimization of the number of sensitive population: 
Set the number of sensitive population around each arc as $r_{3(i, j)}, r_{3}(i, j)=m_{i j} n_{i j}$, there are

$$
\mathrm{W} 3=\min \sum_{i=1}^{n} \sum_{j=1}^{n} r_{3}(i, j)
$$

Along with:

$$
\begin{gathered}
\sum_{(i, k) \in A} X_{i k}-\sum_{(k, i) \in A} X_{k i}= \begin{cases}+1, & \text { i is the beginning point. } \\
-1, & \text { i is the ending point. } \\
0, & \text { otherwise. }\end{cases} \\
X_{i j}^{C} \leq Y_{i j}^{m(c)},(i, j) \in A, c \in C . \\
\sum_{l \in L} p_{i j} r_{i j}^{l} \leq a .
\end{gathered}
$$

In this model, $X_{i j}$ represents whether there is a transport mission on the arc $(\mathrm{i}, \mathrm{j}) ; Y_{i j}$ represents whether the 1,6-Hexanediaminere allowed transport at arc $(\mathrm{i}, \mathrm{j}) ; C_{i j}$ represents accident consequences for arc $(\mathrm{i}, \mathrm{j}) ; h_{i j}$ represents emergency response efficient for arc $(\mathrm{i}, \mathrm{j})$; $P_{i j}$ represents accident rates for $\operatorname{arc}(\mathrm{i}, \mathrm{j}) ; d_{i j}$ represents response length of $\operatorname{arc}(\mathrm{i}, \mathrm{j}) ; v$ represents transportation speed; $m_{i j}$ represents population density of link (i,j); $n_{i j}$ represents sensitive coefficient of the accident; $\alpha$ represents maximum risk limit in the arc (i,j); eqn (3) aims to minimize the accidental risk. Eqn (4) aims to minimize the transportation costs. Eqn (5) aims to minimize the number of sensitive population. Eqn (6) aims to ensure the directional and logistics balance of vehicle transportation. Eqn (7) aims to ensure legal transportation along the path. Eqn (8) aims to establish a maximum risk limit along the path for the government.

\section{SOLVING STEPS OF THE HAZARDOUS CHEMICALS PATH OPTIMIZATION MODEL}

In this paper, proposed optimization algorithm only needs to identify several Pareto optimal solutions and make a comparative analysis for the optimized route. The flow chart for the multi-objective decision model is presented in Fig. 2. The specific steps are as follows.

Step 1: Use the Dijkstra algorithm to find the shortest path for each objective function, respectively. If the shortest paths of the objective function are same, the shortest path is the optimal path of transportation. Otherwise, move to Step 2.

Step 2: Find other objective values for the shortest path of each objective function. Use the three objective values to determine the upper $\mathrm{r}+$ and lower $\mathrm{r}-$.

Step 3: Determine the membership function [13], in eqn (9), $r_{k}^{+}, r_{k}^{-}, r_{k}$ represents upper boundary, lower boundary and the actual weight of the objective function $\mathrm{k}$, respectively.

Step 4: Use an analytic hierarchy process to determine the weight $\mathrm{w}=(\mathrm{w} 1, \mathrm{w} 2, \mathrm{w} 3)$ of each objective function. Select the appropriate weighted square power multiplier $M_{w}^{\beta}$ to represent the objective function in the multi-objective decision model for the dangerous road transportation path as a single objective function. From a practical standpoint, the most important parameters $\beta$ is 1,2 and $-\infty, \beta=1, \beta=2, \beta=-\infty$ represents the weighted arithmetic mean, the weighted quadratic mean and the conjunctive mean, respectively. The computational formula is in eqn (10): 

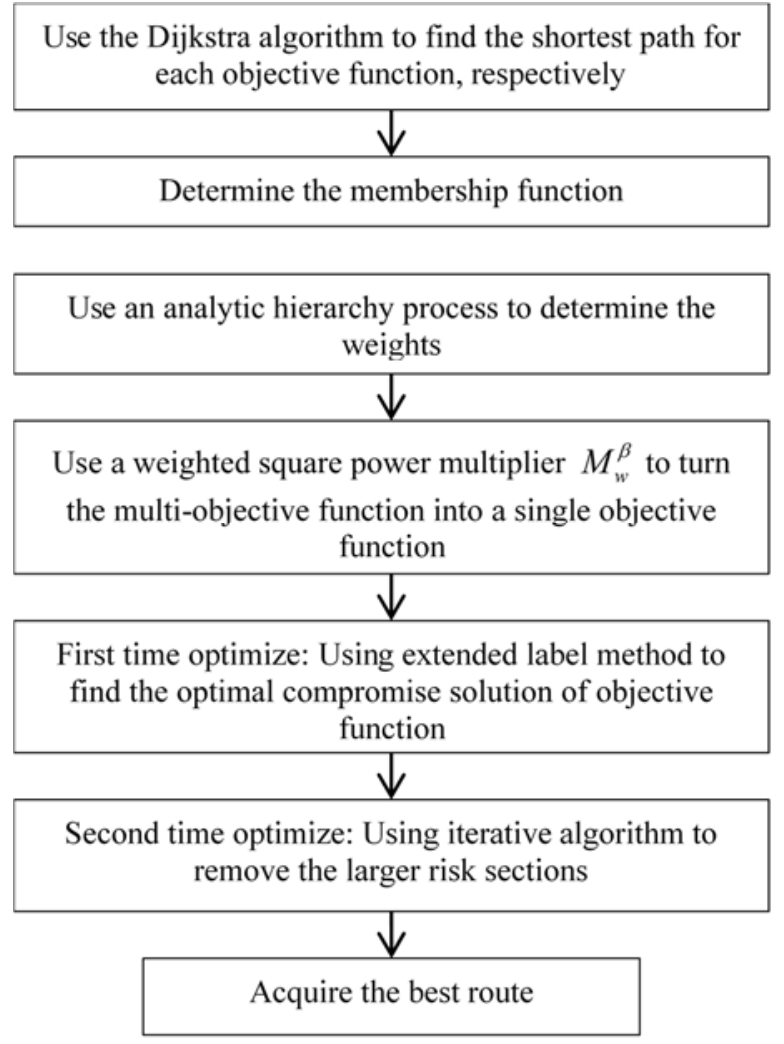

Figure 2: Flow chart of multi-objective decision-making model.

$$
\begin{aligned}
& Z_{k}\left(r_{k}\right)=\left\{\begin{array}{cc}
1, & r_{k} \leq r_{k}^{-} . \\
\frac{r_{k}^{+}-r_{k}}{r_{k}^{+}-r_{k}^{-}}, & r_{k}^{-} \prec r_{k} \prec r_{k}^{+} . \\
0, & r_{k} \geq r_{k}^{+} .
\end{array}\right. \\
& \mu\left(x_{s j}\right)=\left(w_{1}\left(\frac{r_{k 1}^{+}-r_{k 1}}{r_{k 1}^{+}-r_{k 1}^{-}}\right)^{\beta}+w_{2}\left(\frac{r_{k 2}^{+}-r_{k 2}}{r_{k 2}^{+}-r_{k 2}^{-}}\right)^{\beta}+w_{3}\left(\frac{r_{k 3}^{+}-r_{k 3}}{r_{k 3}^{+}-r_{k 3}^{-}}\right)^{\beta}\right)^{\frac{1}{\beta}} .
\end{aligned}
$$

Step 5: Use the extended label method based on the optimal compromised path of the integrated objective function to obtain the optimal compromise solution for dangerous road transportation. This gives the first optimized transportation path. Extended label method [14] is that each node can have many labels, but labels cannot be produced until a path to the label is found. The number of labels owned by a node in a particular cycle is equivalent to the number of paths with a specific capacity from the starting point to the node. 
Step 6: Use the iterative algorithm to remove the larger risk sections, make secondary optimization to the paths, and obtain the optimization path of dangerous chemicals transportation.

\section{EXAMPLE ANALYSIS}

In PDS (Pingdingshan), Henan Province, China, there is a small transport network of dangerous chemicals, which is shown in Fig. 3, and the simplified transportation networks is shown in Fig. 4. Node 1 represents the Zhengshun Chemical Co. Ltd., node 2 represents Pingmei Shenma Chemical Co. Ltd., node 8 represents Suheng Chemical Co. Ltd., node 9 represents Chemical Industry Cluster District, the remaining nodes represents major turning point during the transportation process, the arcs between each node represents roads. The date of distance, accident rate, population etc. of the road in the transportation network was collected and quantified, based on which the weight of transportation risks $r_{1(i, j)}$, transportation costs $r_{2(i, j)}$ and sensitive objective population $r_{3(i, j)}$ of each road can be obtained, as shown in Table 1. There are two kinds of HAZMATs namely 1,6Hexanediamine and Adipic acid that need to be transported from Zhengshun Chemical Co. Ltd. (node 1). to Suheng Chemical Co. Ltd. (node 8) and from Pingmei Shenma Chemical Co. Ltd. (node 2) to Chemical Industry Cluster District (node 9), respectively. In order to meet the requirements of transporters and social groups for transport risks, transport costs and sensitive objective population, we optimize the transportation routes for 1,6Hexanediamine and Adipic acid on the basis of the previous sections.

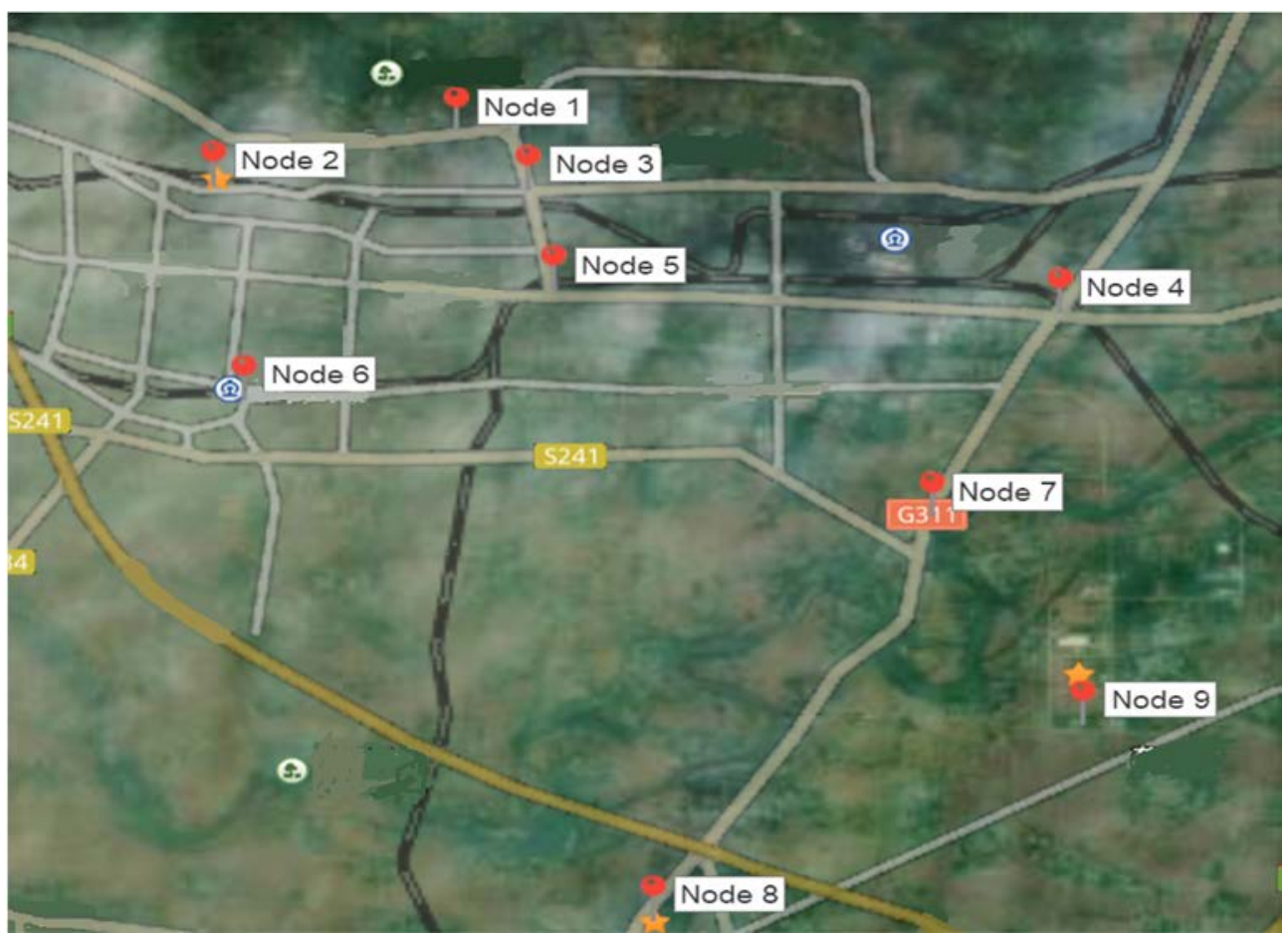

Figure 3: Transportation network of PDS. 


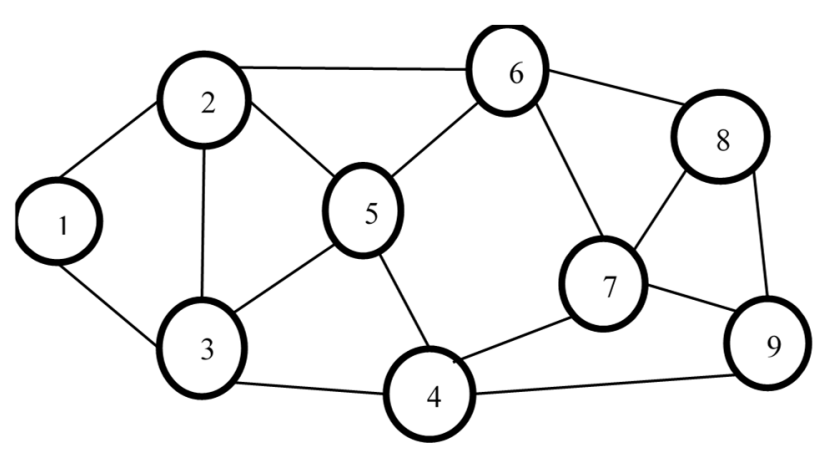

Figure 4: Abstract transportation network.

Table 1: The weights of transport risk, transport cost and sensitive objective population for each arc.

\begin{tabular}{|c|c|c|c|c|c|c|}
\hline & \multicolumn{3}{|c|}{ 1,6-Hexanediamine } & \multicolumn{3}{c|}{ Adipic acid } \\
\hline & $r_{1(i, j)}$ & $r_{2(i, j)}$ & $r_{3(i, j)}$ & $r_{1(i, j)}$ & $r_{2(i, j)}$ & $r_{3(i, j)}$ \\
\hline$(1,2)$ & 5 & 2 & 4 & 7 & 2 & 5 \\
\hline$(1,3)$ & 4 & 1 & 4 & 5 & 1 & 4 \\
\hline$(2,3)$ & 7 & 2 & 5 & 6 & 3 & 5 \\
\hline$(2,5)$ & 8 & 3 & 7 & 7 & 3 & 5 \\
\hline$(2,6)$ & 7 & 3 & 9 & 9 & 6 & 8 \\
\hline$(3,4)$ & 5 & 7 & 5 & 4 & 5 & 4 \\
\hline$(3,5)$ & 4 & 2 & 5 & 4 & 1 & 8 \\
\hline$(4,5)$ & 6 & 6 & 7 & 1 & 1 & 1 \\
\hline$(4,7)$ & 3 & 5 & 3 & 4 & 5 & 6 \\
\hline$(4,9)$ & 7 & 6 & 4 & 7 & 7 & 4 \\
\hline$(5,6)$ & 3 & 4 & 6 & 7 & 4 & 6 \\
\hline$(6,7)$ & 5 & 7 & 4 & 2 & 6 & 5 \\
\hline$(6,8)$ & 3 & 8 & 3 & 4 & 7 & 3 \\
\hline$(7,8)$ & 1 & 7 & 3 & 2 & 5 & 4 \\
\hline$(7,9)$ & 4 & 5 & 3 & 3 & 5 & 3 \\
\hline$(8,9)$ & 5 & 6 & 4 & 5 & 7 & 4 \\
\hline
\end{tabular}

The steps to find the optimal routes for 1,6-Hexanediaminere and Adipic acid are as follows:

1. Find the minimum value for the transportation risk, transportation cost and sensitive population of the arc connected with the origin. The minimum value of 1,6Hexanediamine is 4, 1 and 4, respectively. The minimum value of Adipic acid is 5, 1 and 4 , respectively.

2. Use the Dijkstra algorithm to acquire the shortest paths of each objective function. The shortest paths for 1,6-Hexanediamine are 1-3-4-7-8, 1-2-6-8, and 1-3-4-7-8. The shortest paths for Adipic acid are 2-6-7-9, 2-3-4-9, and 2-3-4-9. 
3. Find two other objective values for the shortest path of each objective function.

4. Ensure the upper bound $r+$ and lower bound $r-$ of the objective function, as shown in Table 2.

5. Ensure the membership function for each goal as follows:

For 1,6-Hexanediamine:

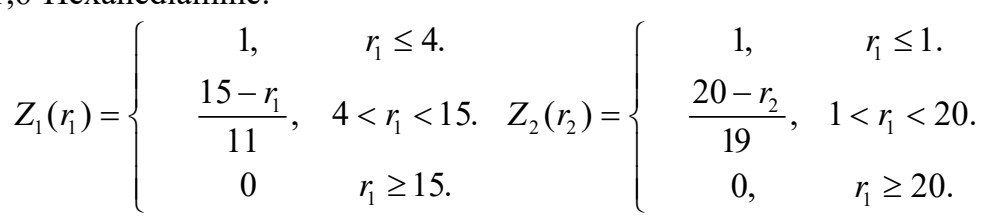

For Adipic acid:

$$
Z_{3}\left(r_{3}\right)=\left\{\begin{array}{cc}
1, & r_{1} \leq 4 \\
\frac{16-r_{3}}{12}, & 4<r_{1}<16 \\
0, & r_{1} \geq 16
\end{array}\right.
$$

$$
\begin{aligned}
& Z_{1}^{*}\left(r_{1}\right)=\left\{\begin{array}{ccc}
1, & r_{1} \leq 5 . \\
\frac{17-r_{1}}{12}, & 5<r_{1}<17 . \\
0, & r_{1} \geq 17 .
\end{array} Z_{2}^{*}\left(r_{1}\right)=\left\{\begin{array}{cc}
1, & r_{1} \leq 1 . \\
\frac{17-r_{1}}{16}, & 1 \prec r_{1} \prec 17 . \\
0, & r_{1} \geq 17 .
\end{array}\right.\right. \\
& Z_{3}^{*}\left(r_{1}\right)=\left\{\begin{array}{cc}
1, & r_{1} \leq 4 \\
\frac{16-r_{1}}{12}, & 4<r_{1}<16 . \\
0, & r_{1} \geq 16
\end{array}\right.
\end{aligned}
$$

6. Determine the weight of each objective for the two types of Adipic acidy Analytic Hierarchy Process (AHP), finding that the weight of 1,6-Hexanediamine is $\mathrm{W}=(\mathrm{w} 1, \mathrm{w} 2$, $\mathrm{w} 3)=(0.1046,0.6089,0.2865)$ and the weight of Adipic acid is $\mathrm{W}^{*}=\left(\mathrm{w}^{*} 1, \mathrm{w}^{*} 2\right.$, $\left.\mathrm{w}^{*} 3\right)=(0.667,0.222,0.111)$.

7. Select the appropriate weighted square power factor $M_{w}^{(\beta)}$ and combine it with the membership function to convert the multi-objective shortest path problem into the maximized fuzzy trade-off problem. Next, use the extended label correcting method to find the most satisfactory paths. Under the established conditions of the most satisfactory paths, give the paths of transportation risk, transportation cost and sensitive population. When $\beta=1,2,-\propto$, it can be calculated according to eqn (10).

8. First time optimization. Making MATLAB program to execute the above algorithm, the results of the program can be obtained in Table 3 . The most satisfactory transport routes of 1,6-Hexanediamine are 1-2-6-8, and the most satisfactory transport routes of Adipic acid is 2-3-4-9 and 2-6-8-9.

Table 2: Upper boundary $r+$ and lower boundary $r-$ of the objective functions.

\begin{tabular}{|l|c|c|}
\hline & $\begin{array}{c}(\mathrm{r}+, \mathrm{r}-) \text { of } 1,6- \\
\text { Hexanediamine }\end{array}$ & $(\mathrm{r}+, \mathrm{r}-)$ of Adipic acid \\
\hline Transportation risk & $(15,4)$ & $(17,5)$ \\
\hline Transportation cost & $(20,1)$ & $(17,1)$ \\
\hline Sensitive population & $(16,4)$ & $(16,4)$ \\
\hline
\end{tabular}


Table 3: Results for the first optimization.

\begin{tabular}{|l|c|c|c|c|c|c|}
\hline & $\mathrm{w}$ & $\beta$ & $\begin{array}{c}\text { The best } \\
\text { route }\end{array}$ & $\begin{array}{c}\text { Transportation } \\
\text { risk }\end{array}$ & $\begin{array}{c}\text { Transportation } \\
\text { cost }\end{array}$ & $\begin{array}{c}\text { Sensitive } \\
\text { population }\end{array}$ \\
\hline \multirow{3}{*}{ 1,6-Hexanediamine } & $(0.1046$, & 1 & $1-2-6-8$ & 15 & 13 & 16 \\
\cline { 3 - 7 } & 0.6089, & 2 & $1-2-6-8$ & 15 & 13 & 16 \\
\cline { 3 - 7 } & $0.2865)$ & -0 & $1-2-6-8$ & 15 & 13 & 16 \\
\hline \multirow{3}{*}{ Adipic acid } & $(0.667$, & 1 & $2-3-4-9$ & 17 & 15 & 13 \\
\cline { 3 - 7 } & 0.222, & 2 & $2-6-8-9$ & 18 & 20 & 15 \\
\cline { 3 - 7 } & $0.111)$ & -9 & $2-3-4-9$ & 17 & 15 & 13 \\
\hline
\end{tabular}

Table 4: Corresponding costs and risks for the shortest routes for transportation cost.

\begin{tabular}{|l|c|c|c|c|}
\hline & Route & $\begin{array}{c}\text { Transportation } \\
\text { cost }\end{array}$ & $\begin{array}{c}\text { Transportation } \\
\text { risk }\end{array}$ \\
\hline 1,6-Hexanediamine & Route1-11 & $1-2-6-8$ & 13 & 15 \\
\hline Adipic acid & Route2-11 & $2-3-4-9$ & 15 & 17 \\
\hline
\end{tabular}

9. Use the iterative algorithm to optimize the routes that are obtained above. The flow chart of the iterative algorithm is shown in Fig. 5.

1) First iteration:

1-1) Upper path selection: as shown in the step (2), the minimum risk routes for 1,6Hexanediamine and Adipic acid are 1-3-4-7-8 and 2-6-7-9, respectively. While in step (8), the minimum risk routes are 1-2-6-8 and 2-3-4-9, respectively. Therefore, the upper transportation network can be obtained, as shown in Fig. 6(a). As well, the corresponding risk value is $\mathrm{R} 11=13$ and $\mathrm{R} 21=14$, and the total risk value $\mathrm{R} 1=27$.

1-2) Lower path selection: based on Fig. 6(a), the shortest cost paths are Route1-11: 1-2- 6-8 and Route2-11: 2-3-4-9. The corresponding costs and risks are shown in Table 4, and the total cost value $\mathrm{C} 1=28, \mathrm{R}^{\prime} 1=32$.

1-3) Stability judgment: from $1-1$ and $1-2$, it can be seen that when $\mathrm{R}^{\prime} 1=32, \mathrm{R}^{\prime} 1 \neq R 1$ and $\frac{R^{\prime} 1-R 1}{R 1} \leq 10^{-5}$ is not satisfied. Therefore, the upper layer network is unstable.

1-4) Feedback adjustment: according to the feedbacks of road users, a certain section of road 6-7 has been used for driving test, which may have many uncertainties. Besides, compared with other transport roads, the transportation cost of road 6-7 is higher. Therefore, considering these feedbacks, the road 6-7 is removed from Fig. 6(a), and a new transportation network is obtained, as shown in Fig. 6(b).

2) Second iteration:

According to the second iteration based on the first iteration, the optimization routes 1-26-8 and 2-3-4-9 are obtained. They not only meet the extended label correcting method, but also satisfy the stability test of the iterative algorithm. In addition, according to the statistics of the relevant departments of PDS, the traffic conditions of the road 1-2-6-8 and 2-3-4-9 are better than other roads. When transporting on these roads, the transport risks, transport costs and sensitive objective population can better meet the requirements of transporters and social groups. Therefore, the optimal transport routes of the 1,6-Hexanediamine and Adipic acid are 1-2-6-8 and 2-3-4-9, respectively. The final optimal route results are shown in Fig. 7. 


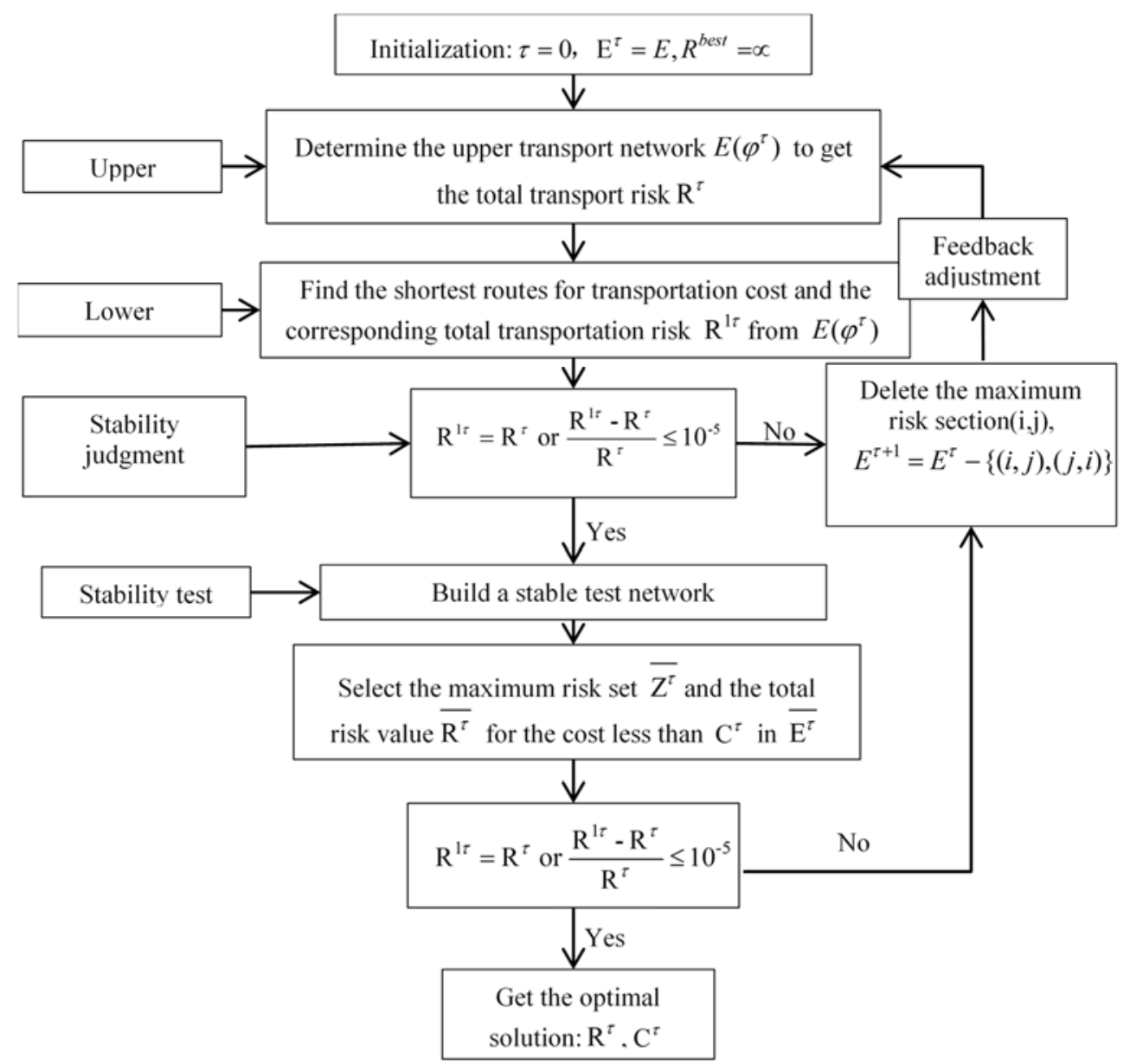

Figure 5: Flow chart of the iterative algorithm.

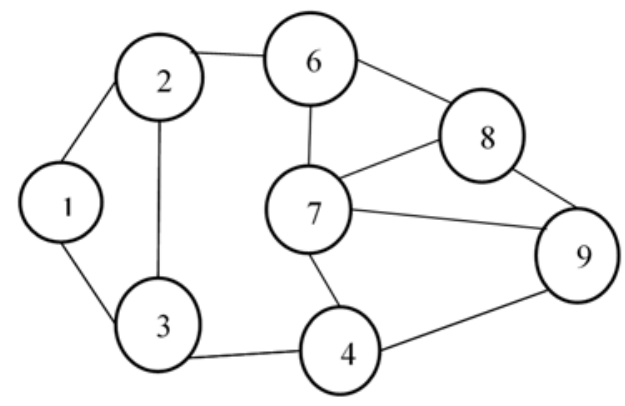

(a)

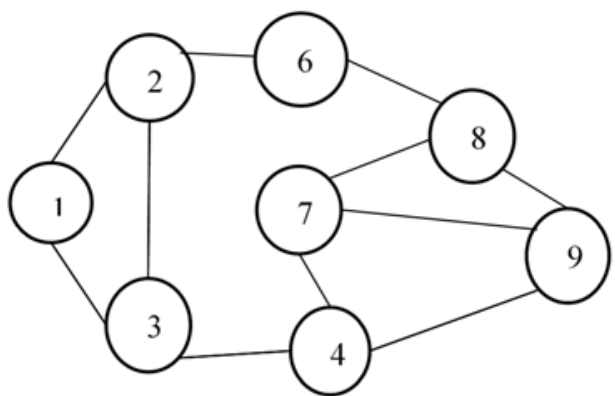

(b)

Figure 6: (a) The upper transport network; (b) The lower transportation network. 


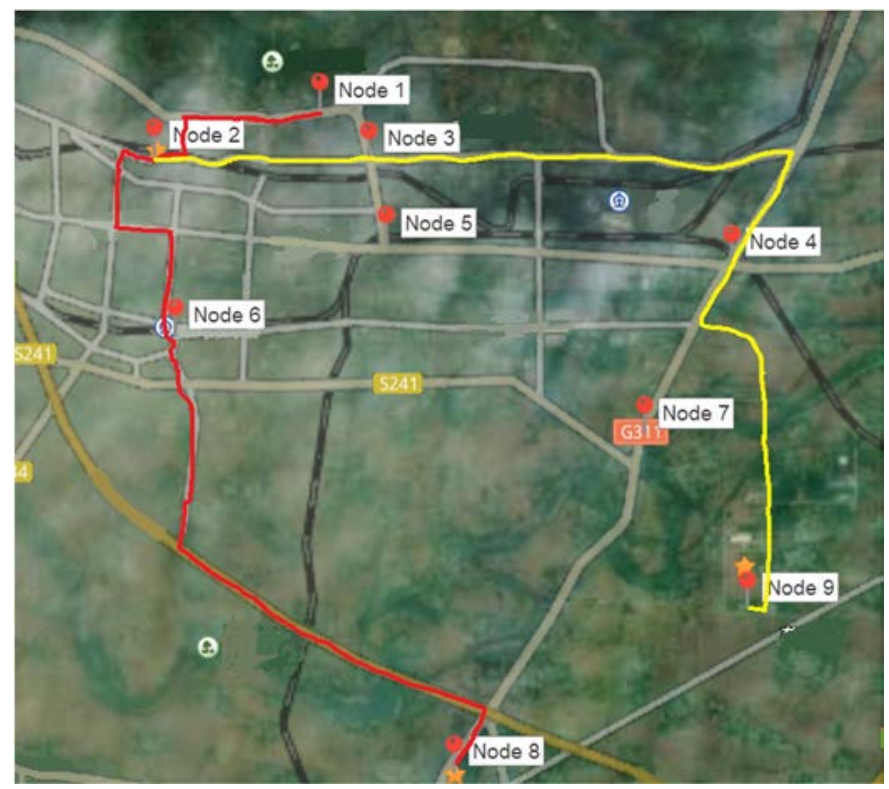

Figure 7: Optimal road map of 1,6-Hexanediamine (the red line) and Adipic acid (the yellow line).

\section{CONCLUSIONS AND FUTURE WORK}

In this paper, we proposed a path optimization model integrated risk decision for dangerous chemical transportation on the basis of an iteration algorithm and extended label correcting method, where we simultaneously optimize three objective functions. Under the premise of comprehensive use of multiple optimization methods, the proposed model takes the multipoint and multi-objective transportation in the actual transport process into account. Firstly, the weight vector of objective functions of the transport network is solved by the risk decision model namely analytic hierarchy process (AHP). Then, the transport network is optimized by the extended label correcting method. Finally, on the basis of the extended label correcting method, iterative algorithm is used to re-optimize and get the best optimization route. Compared with the traditional one-off path optimization, the optimization model proposed in this paper, takes into account the multi-point and multi-objective transportation of various dangerous chemicals, and compensates for the shortcomings of the one-off optimization method, so that make the final optimization path the best one to meet the needs of concerned aspects.

A transport case study of two HAZMATs transportation is analysed to demonstrate the proposed model and solutions. From the results of the case study, it is found that the proposed algorithm meets the requirements of transportation risk, cost and sensitive populations, and overall arrives at a more reasonable optimization path. The results obtained also show that using the proposed comprehensive optimization method to optimize the HAZMAT transportation route can help decision makers better choose the optimal route.

With the rapid development of chemical enterprises, the demand for hazardous chemicals is on the rise, and the danger to people's property and life caused by hazardous chemicals in the transportation process is becoming more and more serious. In order to minimize the negative impact on people, the research on the transportation of specific hazardous chemicals, 
the setting of transport target and the method of route optimization of hazardous chemicals are the future research directions.

\section{ACKNOWLEDGEMENTS}

This work was supported by the National Key Research and Development Program of China (2016YFC0801500), the National Natural Science Foundations of China (No. 21406115 and No.51606092), and a project funded by the Priority Academic Program Development of Jiangsu Higher Education Institutions. The authors deeply appreciate their supports.

\section{REFERENCES}

[1] Xing, G.Z., Analysis of risk capacity of dangerous goods transportation in chemical industry park. Chemical Enterprise Management, p. 132, 2016.

[2] United Nations Economic Commission for Europe, Recommendations on the Transport of Dangerous Goods. ST/ST/AC/1/REV, 19(1), pp. 249-254, 2009.

[3] Bianco, L., Caramia, M., Giordani, S. \& Piccialli, V., Operations Research Models for Global Route Planning in Hazardous Material Transportation. Handbook of OR/MS Models in Hazardous Materials Transportation, eds R. Batta \& C. Kwon, Springer: New York, pp. 49-101, 2013.

[4] Tavakkoli-Moghaddam, R., Azarkish, M. \& Sadeghnejad-Barkousaraie, A., Solving a multi-objective job shop scheduling problem with sequence-dependent setup times by a Pareto archive PSO combined with genetic operators and VNS. International Journal of Advanced Manufacturing Technology, 53(5-8), pp. 733-750, 2011.

[5] Frank, W.C., Thill, J.C. \& Batta, R., Spatial decision support system for hazardous material truck routing. Transportation Research Part C, 8(1-6), pp. 337-359, 2000.

[6] Clegg, J., Smith, M., Xiang, Y. \& Yarrow, R., Bilevel programming applied to optimising urban transportation. Transportation Research Part B Methodological, 35(1), pp. 41-70, 2001.

[7] Samanlioglu, F., A multi-objective mathematical model for the industrial hazardous waste location-routing problem. European Journal of Operational Research, 226(2), pp. 332-340, 2013.

[8] Erkut, E. \& Gzara, F., Solving the hazmat transport network design problem. Computers \& Operations Research, 35(7), pp. 2234-2247, 2008.

[9] Bianco, L., Caramia, M. \& Giordani, S., A bilevel flow model for hazmat transportation network design. Transportation Research Part $C$ Emerging Technologies, 17(2), pp. 175-196, 2009.

[10] Kara, B.Y. \& Verter, V., Designing a road network for hazardous materials transportation. Transportation Science, 38(2), pp. 188-196, 2004.

[11] Ma, C.X., Guang, X.P., Wu, F. \& Zhao, Y.C., Highway transportation route decisionmaking of hazardous material in developed transportation network. Journal of Transportation Systems Engineering \& Information Technology, 9(4), pp. 134-139, 2009.

[12] Zografos, K.G. \& Davis, C.F., Multi-objective programming approach for routing hazardous materials. Journal of Transportation Engineering, 115(6), pp. 661-673, 1989.

[13] Han, S.L. \& Li, H.X., Fuzzy programming approach solution for multi-objective solid transportation problem. Journal of Southeast University (English edn), 20(1), pp. 102107, 2004.

[14] Skriver, A.J.V. \& Andersen, K.A., A label correcting approach for solving bicriterion shortest-path problems. Computers \& Operations Research, 27(6), pp. 507-524, 2000. 